\title{
Population Burden of Long-Term Survivorship After Severe Sepsis in Older Americans
}

\author{
Theodore J. Iwashyna, MD, PhD, ${ }^{* \dagger}$ Colin R. Cooke, MD, ** Hannah Wunsch, MD, ${ }^{\xi \|}$ and \\ Jeremy M. Kahn, MD**t
}

OBJECTIVES: To ascertain the absolute number of Medicare beneficiaries surviving at least 3 years after severe sepsis and to estimate their burden of cognitive dysfunction and disability.

DESIGN: Retrospective cohort analysis of Medicare data.

SETTING: All short-stay inpatient hospitals in the United States, 1996 to 2008.

PARTICIPANTS: Individuals aged 65 and older.

MEASUREMENTS: Severe sepsis was detected using a standard administrative definition. Case-fatality, prevalence, and incidence rates were calculated.

RESULTS: Six hundred thirty-seven thousand eight hundred sixty-seven Medicare beneficiaries were alive at the end of 2008 who had survived severe sepsis 3 or more years earlier. An estimated 476,862 (95\% confidence interval $(\mathrm{CI})=455,026-498,698)$ had functional disability, with $106,311(95 \% \mathrm{CI}=79,692-133,930)$ survivors having moderate to severe cognitive impairment. The annual number of new 3-year survivors after severe sepsis rose $119 \%$ during 1998 to 2008 . The increase in survivorship resulted from more new diagnoses of severe sepsis rather than a change in case-fatality rates; severe sepsis rates rose from 13.0 per 1,000 Medicare beneficiary-years to 25.8 $(P<.001)$, whereas 3-year case fatality rates changed much less, from $73.5 \%$ to $71.3 \%(P<.001)$ for the same cohort. Increasing rates of organ dysfunction in hospitalized individuals drove the increase in severe sepsis inci-

From the "Department of Medicine, University of Michigan, Ann Arbor, Michigan; ${ }^{\dagger}$ Center for Clinical Management Research, Ann Arbor Veterans Affairs Health Services Research and Development Service Center of Excellence, Ann Arbor, Michigan; ${ }^{\dagger}$ Robert Wood Johnson Clinical Scholars Program, University of Michigan, Ann Arbor, Michigan; ${ }^{\S}$ Departments of Anesthesiology, " Epidemiology, Columbia University, New York, New York; **Clinical Research, Investigation and Systems Modeling of Acute Illness Center, Department of Critical Care Medicine, School of Medicine, and ${ }^{\dagger}$ Department of Health Policy and Management, Graduate School of Public Health, University of Pittsburgh, Pittsburgh, Pennsylvania.

Address correspondence to Theodore J. Iwashyna, 3 a23 300 NIB, SPC 5419, 300 North Ingalls, Ann Arbor, MI 48109. E-mail:

tiwashyn@umich.edu

DOI: 10.1111/j.1532-5415.2012.03989.x dence, with an additional small contribution from population aging.

CONCLUSIONS: Sepsis survivorship, which has substantial long-term morbidity, is a common and rapidly growing public health problem for older Americans. There has been little change in long-term case-fatality, despite changes in practice. Clinicians should anticipate more-frequent sequelae of severe sepsis in their patient populations. J Am Geriatr Soc 60:1070-1077, 2012.

Key words: Medicare; severe sepsis; disability; population health

Cevere sepsis occurs when an acute infection leads to $\checkmark$ organ dysfunction. ${ }^{1}$ This syndrome encompasses many common causes of hospitalization, such as pneumonia with hypoxemia or urinary tract infection complicated by acute renal failure. Severe sepsis is also a common cause of critical illness, as when the organ dysfunctions include acute respiratory failure or shock. Severe sepsis has been recognized to have a high acute risk of death. ${ }^{2}$ Morerecent data demonstrate that survivors of severe sepsis-a majority of those with the diagnosis - have poor quality of life, ${ }^{3,4}$ frequently develop cognitive and functional disability, ${ }^{5}$ and require substantial ongoing acute and long-term care. $^{6,7}$ These levels of disability impose a substantial burden on caregivers. ${ }^{8}$ Severe sepsis has been termed "a quintessential disease of aged." 9

Although the effect of severe sepsis on individuals is understood, little is known about the effect of severe sepsis on population health-particularly the effect on population levels of disability. This stands in marked contrast to conditions such as cancer and stroke, of which the burdens of survivorship are considered core components of patient management and effect on public health. ${ }^{10}$ Physicians caring for individuals who have survived cancer are aware of the special physical and emotional sequelae for which they must maintain a high index of suspicion. ${ }^{11,12}$ No such 
information exists about whether the population burdens of severe sepsis survivorship warrant consideration in discussions of population health, disability, and caregiving needs.

Therefore, the current study sought to measure the incidence and prevalence of long-term survivorship after severe sepsis in Medicare beneficiaries. The primary objectives were to measure the absolute size of the population Medicare beneficiaries who survive at least 3 years after severe sepsis and to estimate, based on prior work, ${ }^{5}$ the likely numbers with cognitive dysfunction and disability. Temporal trends in survivorship were also examined, and several targeted hypotheses regarding potential changes in sepsis survivorship over time were tested. Specifically, it was hypothesized that changes in sepsis survivorship might have resulted from the aging of the population, changing age-specific rates of hospitalization with infection, changing rates of acute organ dysfunction in those with infections, or changes in the case-fatality rate in those with severe sepsis.

\section{METHODS}

\section{Definition of Survivorship}

Conceptually, an individual is a "survivor" when the acute burdens of an illness have passed but the sequelae of that illness and its treatment may now become important to his or her health and functioning. ${ }^{13}$ Operationalizing this is typically done by defining a time point-necessarily somewhat arbitrary-and examining those who are alive at least that long after diagnosis. ${ }^{10}$ The primary outcome of the current study was survival to 3 years from date of admission, which is termed 3-year survivorship; this was based on clinical experience, data on the period of the greatest risk of mortality after severe sepsis, and comparability with other reports. ${ }^{14,15}$

\section{Data Source}

This retrospective cohort study analyzed all fee-for-service Medicare beneficiaries aged 65 and older in the 1996 to 2008 Medicare Provider Analysis and Review (MedPAR) files and the linked Medicare Denominator Files. Medicare provides insurance for more than $96 \%$ of older Americans. ${ }^{16}$ Fewer than $15 \%$ of Medicare beneficiaries were in Medicare managed care programs that did not file claims; such patients were excluded from these analyses.

A previously validated and widely used claims-based definition of severe sepsis was relied on..$^{2,6,7,17-21}$ Only inpatient hospitalizations in short-stay hospitals (as opposed to long-term acute care or skilled nursing facilities) were eligible. This definition requires evidence of an infection and new-onset organ dysfunction during a single hospitalization, in accordance with the internationally accepted consensus definition of severe sepsis, ${ }^{1,22}$ and in validation studies identifies a similar population to those identified through chart review. ${ }^{23}$ This definition has prognostic validity, identifying individuals with substantial short- ${ }^{2}$ and long-term mortality, ${ }^{5}$ high healthcare-related spending, ${ }^{6,7}$ and high risk of long-term cognitive and physical disability. ${ }^{5}$ This definition is distinct from other epidemiological approaches to the study of sepsis (rather than severe sepsis) that primarily use codes for explicit septicemia, bacteremia, and disseminated fungal infections rather than for all infections. ${ }^{24}$ The components of the severe sepsis definition were used to define hospitalizations with infection as well as organ dysfunctions. A code for "severe sepsis" or "septic shock" was considered to be evidence of at least one organ dysfunction, according to the consensus definition.

As a secondary outcome, spending was defined as the amount Medicare reimbursed the hospital for the inpatient stay. To provide a point of comparison for the sepsis findings, the incidence and costs of acute myocardial infarction (AMI) as a common cause of critical illness of established importance to population health were also examined. Hospitalizations with AMI were defined as those with a primary International Classification of Diseases, Ninth Revision, Clinical Modification (ICD-9-CM) diagnostic code of 410.xx (excluding 410.x2). ${ }^{25,26}$ Cases with a length of stay of 1 day were excluded - unless that patient died, left against medical advice, or was transferred to another hospital-because such a short length of stay probably represented exclusion admissions and not true AMI. $^{27}$

\section{Disability and Cognitive Impairment Definitions}

The burdens of moderate to severe cognitive impairment and functional disability were estimated using previously published data for individuals surviving 3 years after severe sepsis using the same definition of severe sepsis and drawn from a similar population of Medicare beneficiaries. ${ }^{5}$ In that study, individuals or their proxies were asked whether, as a result of a health problem, they required assistance with any of six activities of daily living (ADLs: walking, dressing, bathing, eating, getting into and out of bed, and toileting) or five instrumental activities of daily living (IADLs: preparing a hot meal, shopping for groceries, making telephone calls, taking medicines, and managing money). Disability was defined as a limitation in any ADL or IADL due to a health problem.

Cognitive impairment was defined as poor performance on the modified Telephone Interview for Cognitive Status (m-TICS) or cognitive problems as reported by a proxy informant similar to a level of impairment associated with dementia. The m-TICS is 35-point scale that includes tests of memory, serial seven subtractions, naming, and orientation, with a score of 0 to 7 defining moderate to severe cognitive impairment. ${ }^{28,29}$ For individuals who were unable to be interviewed themselves, the validated Informant Questionnaire on Cognitive Decline in the Elderly ${ }^{30}$ was administered to proxies, and a score of 4.59 to 5.00 indicated moderate to serve cognitive impairment.

Using these definitions in the population of individuals alive 3 years after severe sepsis in the Health and Retirement Study, ${ }^{5}$ it was shown that the prevalence of moderate to severe cognitive impairment was $16.7 \%$ (95\% confidence interval $(\mathrm{CI})=12.3-21.0 \%)$, and $74.8 \% \quad(95 \%$ $\mathrm{CI}=71.3-78.2 \%$ ) of survivors had at least one limitation in an ADL or IADL. This disability includes that which existed before severe sepsis and that associated with severe sepsis. 


\section{Analysis}

The primary outcomes, the incidence and prevalence of severe sepsis survivorship, were calculated in fee-for-service Medicare beneficiaries.

To examine temporal trends in the primary outcome, standard demographic methods were used to measure the relative contributions when many contributing rates were each changing. The number of survivors of severe sepsis was the product of the number of incident cases of severe sepsis multiplied by 1 minus the case fatality rate. The number of incident cases of severe sepsis was the product of the number hospitalizations with infection multiplied by the organ failure rate per hospitalization with infection. The number of hospitalizations with infection was the product of the number of people at risk in each age group multiplied by the age group-specific infection rate. A working example of this approach is provided in Appendix 1.

All rates were calculated on a monthly basis. For numerators and denominators, individuals were classified according to their age on the first of each month. All months in which individuals were in fee-for-service Medicare were analyzed.

To test the sensitivity of the conclusions to changes in specific coding practices for the organ dysfunctions used in the definition, all of the analyses were replicated sequentially excluding each organ system. Detailed results are in Appendix 2.

For statistical comparisons, annual differences in incidence rate ratios were tested for using Stata 10.1 (Stata Corp., College Station, TX). $P<.05$ was considered statistically significant. Given the large numbers of cases, statistical significance should not be equated with clinical significance. The University of Michigan institutional review board approved this work.

\section{RESULTS}

In 1996, 34,782,442 Medicare beneficiaries aged 65 and older were examined for a total of $357,662,059$ beneficiary-months. Their median age was 73 (interquartile range (IQR) 68-79); 59\% were female. In 2008, $39,337,348$ Medicare beneficiaries were examined for a total of $350,267,105$ beneficiary-months in 2008. Their median age was 73 (IQR 68-80); 57\% were female.

\section{Absolute Number of Survivors and Estimated Population-Level Burden of Disability}

As defined, the incidence of 3-year survivorship in a given year is the number of individuals still alive exactly 3 years after being hospitalized with severe sepsis in Medicare. Using this definition, there were 225,251 new 3-year survivors in Medicare in 2008 (hospitalized for severe sepsis in 2005), up from 102,767 new 3-year survivors in 1999 (hospitalized in 1996), an increase of $119 \%$ in a decade (Figure 1). Repeating the analysis for 5-year survival, the numbers of new survivors rose from 67,799 in 2001 (hospitalized in 1996) to 121,029 in 2008 , an increase of $79 \%$.

As defined, the prevalence of 3-year severe sepsis survivorship in a given year in Medicare is the number of

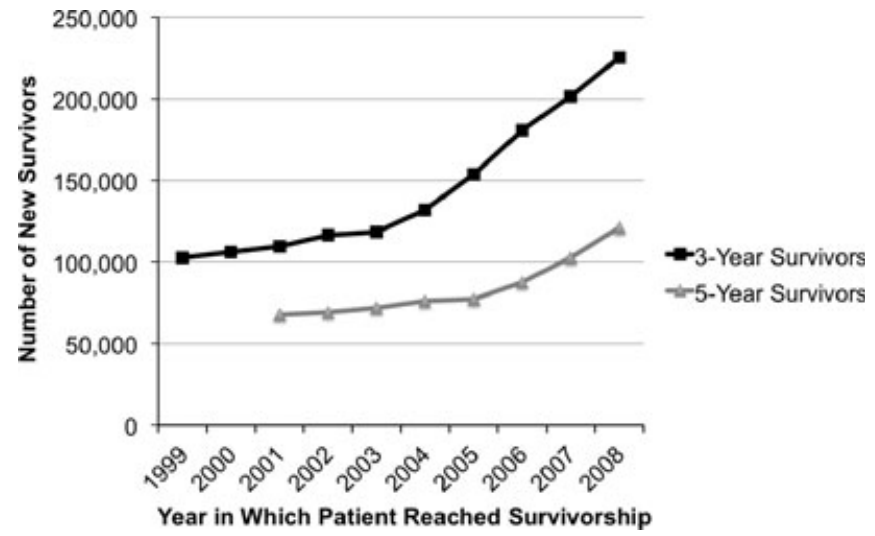

Figure 1. New 3- and 5-year survivors after severe sepsis. See also Figure 4, which shows the relative contributions of increasing rates of organ dysfunction per hospitalization with infection, increasing rates of hospitalization with infection, population aging, and changes in the 3 -year case fatality to these trends.

individuals hospitalized for severe sepsis at least 3 years earlier and still alive. Using this definition, 637,867 individuals had survived severe sepsis by at least 3 years as of the end of 2008 (344,111 who had survived by at least 5 years). This 3 -year survivorship number implies that there were at least 106,311 survivors $(95 \% \mathrm{CI}=79,692$ 133,930 survivors) with moderate to severe cognitive impairment at the end of 2008 in Medicare. In that same population, there were $476,862 \quad(95 \% \quad \mathrm{CI}=455,026-$ $498,698)$ survivors with functional disability, requiring assistance with at least one ADL or IADL.

\section{Changes in Number of Cases of Severe Sepsis}

There was a significant increase in the number of hospitalizations for severe sepsis in Medicare (Figure 2). Whereas 387,330 individuals were hospitalized with severe sepsis in 1996 , by $2008,1,015,432$ were hospitalized. The rates of

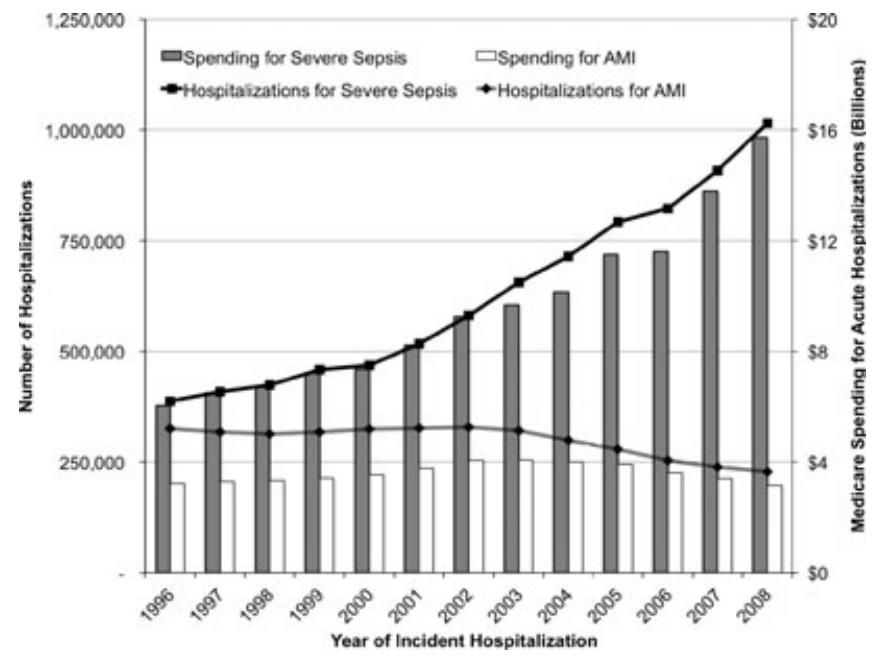

Figure 2. Hospitalizations and spending for severe sepsis and acute myocardial infarction in Medicare. Spending is for the acute hospitalization only and is not inflation adjusted. $\mathrm{AMI}=$ acute myocardial infarction. 
hospitalization for severe sepsis increased from 13.0 per 1,000 Medicare beneficiary-years in 1996 to 34.8 in 2008 $(P<.001)$. An increase in direct Medicare spending on hospitalizations for severe sepsis from $\$ 6.03$ billion to $\$ 15.73$ billion accompanied these rising rates of hospitalization. To contextualize these numbers for severe sepsis, they were contrasted with those seen for AMI (Figure 2). In 1996, there were 325,108 hospitalizations for AMI, an incidence of 10.91 per 1,000 Medicare beneficiary-years, at a cost of $\$ 3.22$ billion. In 2008 , there were 227,298 cases of AMI in Medicare in 2008, an incidence of 7.79 per 1,000 Medicare beneficiary-years, at a cost to Medicare of $\$ 3.16$ billion for the acute hospitalizations.

The rising numbers of hospitalization with severe sepsis resulted primarily from a rise in the rate of organ dysfunction per hospitalization with infection. The absolute number of hospitalizations with infection increased from 108 to 121 per 1,000 Medicare beneficiary-years $(P<.001)$. Rates of organ dysfunction in individuals hospitalized with infection rose more rapidly, from $12.1 \%$ in 1996 to $28.8 \%$ in $2008(P<.001)$. Rates of severe organ dysfunction, defined as the presence of three or more organ dysfunctions, rose from $0.33 \%$ of hospitalizations with infection in 1996 to $1.64 \%$ in 2008, or from $2.7 \%$ of hospitalizations with severe sepsis to $5.7 \%$ of such hospitalizations (both $P<.001$ ).

\section{Changes in Case Fatality Rate}

The potential contribution of changes in case-fatality rate to the rising severe sepsis survivorship is shown in Figure 3. There were small improvements in long-term case-fatality rates after severe sepsis during this period. Three-year case fatality for individuals who developed severe sepsis between 1996 and 2005 fell from $73.5 \%$ to $71.3 \% \quad(P<.001)$. These relatively flat long-term casefatality rates contrast with short-term figures. The inpatient case fatality dropped from $28.5 \%$ in 1996 to $15.8 \%$ in 2008, with more-modest declines seen in patient-centered short-term outcomes such as 30-day case fatality $(32.2 \%$ to $24.9 \%)$ and 90 -day case fatality $(43.9 \%$ to $36.3 \%)$ (all $P<.001)$.

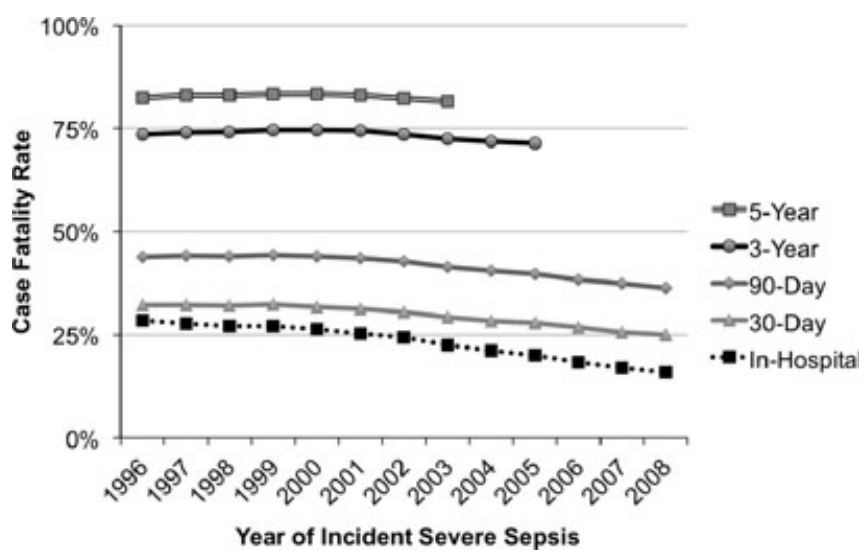

Figure 3. Case-fatality rates after severe sepsis, according to year in which severe sepsis developed. The case-fatality rate is the fraction of hospitalizations after which the individuals died according to the specified time period.

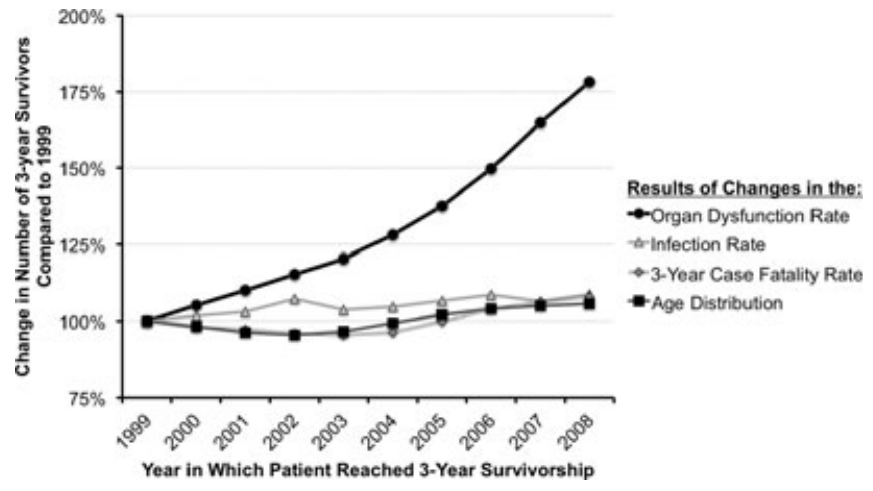

Figure 4. Contributions of the factors to the rising number of survivors of severe sepsis. This figure shows the relative number of 3-year survivors who would have been expected had only each given factor changed. Interpretive example: The aging of the population alone would have resulted in a $5.7 \%$ increase in the number of 3-year survivors between 1999 and 2008 had other rates remained the same. The figure reveals that the most important contributor to the growth in the number of survivors of severe sepsis over this decade was the increase in the rate of organ dysfunction per patient hospitalized with infection, not by better survival among patients with severe sepsis.

\section{Relative Importance of Alternative Mechanisms}

The increase in severe sepsis incidence-particularly the greater incidence of organ dysfunctions in individuals hospitalized with infections-drove the majority of the increase in the number of survivors (Figure 4). Thus 3-year survivorship grew $78.2 \%$ because of the rising rate of organ dysfunctions in individuals hospitalized with infection, whereas the changes in the age distribution, agespecific infection rate, and 3-year case-fatality rate each contributed less than $10 \%$ to the growth in severe sepsis survivors.

\section{Sensitivity Analyses}

Several sensitivity analyses were conducted for the resultsparticularly to changes in coding practice (Appendix 2) — and consistent results were found.

\section{DISCUSSION}

Sepsis survivorship is a common and growing feature of the health care of older Americans. This study establishes for the first time the pervasiveness of a problem already proven to be important to individuals and their families; sepsis survivors are known to be at substantial risk of poor quality of life, functional disability, and cognitive impairment. ${ }^{3,4,25,31}$ Hundreds of thousands of individuals with severe sepsis are surviving years after their illness and face these challenges. These Medicare data show consistently rising rates of incident severe sepsis, consistent with past work in the United States and more-recent work in Europe. ${ }^{24,32}$ This study also demonstrates for the first time that a rising incidence of severe sepsis, rather than changes in the case-fatality rate after severe sepsis, is driving the rise in survivorship. 
These findings constitute a set of challenges to physicians and policy-makers. These data suggest a substantial population burden of older adults with important disability after severe sepsis-more than 100,000 with moderate to severe cognitive impairment and nearly 500,000 with functional disability. These numbers of disabled survivors of severe sepsis are of the same order of magnitude as the numbers of survivors of other recognized public health problems such as breast cancer survivorship. ${ }^{10}$ Moreover, there is evidence that some-perhaps much-of this disability is attributable to severe sepsis, rather than severe sepsis being simply a marker for disability. (From individual-level longitudinal data on severe sepsis survivors, a hospitalization for severe sepsis was associated with 3.34 times greater odds of moderate to severe cognitive impairment. For those with no disability before severe sepsis, such a hospitalization was associated with the development of 1.57 new limitations in ADLs and IADLs, and for those with mild to moderate limitations beforehand, sepsis was associated with the development of 1.50 new limitations. ${ }^{5,19}$ ) Such population burden of disability after severe sepsis argues for an important role of sepsis prevention and disability mitigation in preserving the independence of older Americans. Moreover, the downstream costs of severe sepsis may be substantial - not only in terms of direct healthcare costs from disability, but also in terms of caregiver burden, lost productivity, and informal care needs. Investments by Medicare in programs such as early mobility $^{33}$ and delirium prevention ${ }^{34}$ may offer longterm returns - in better functioning and lower healthcare costs- but this needs to be studied.

From a clinical perspective, the importance of increasing rates of organ dysfunction to rising severe sepsis incidence (and hence total numbers of surviving patients) argues for particular focus on the early inpatient detection and management of severe sepsis. It has been hypothesized that there may be a critical window during which effective treatment of infection (the rates of which are rising only slightly) may prevent the inflammatory and coagulopathic cascade of severe sepsis that leads to organ dysfunction. ${ }^{35}$ If this hypothesis is correct, preventing the conversion of infection to severe sepsis may have a marked influence on the population burden of sepsis survivorship. Interventions such as early antibiotics ${ }^{36}$ and pneumococcal vaccination may be effective in this arena-and the cost-effectiveness analysis should consider their potential to avert the high societal cost of disability after severe sepsis. It may be hypothesized that the rising rates of organ dysfunction result from several causes, including growing use of immunosuppressive medications, ${ }^{37}$ higher thresholds for hospital admission in an era of increasing shift toward outpatient treatment, ${ }^{38}$ and increased diagnosis of organ injury with more-sensitive and ubiquitous testing ${ }^{39}$; establishing the relative contributions of these different mechanisms may be an important topic for future research.

There is little evidence of significant declines in casefatality rates over longer patient-centered time horizons. Cohorts that experienced declines in short-term mortality did not have corresponding declines in long-term mortality, even though sepsis appears to exert a mortality effect for at least 5 years after diagnosis. ${ }^{14,15}$ This unchanged long-term mortality occurred despite the publication of several landmark trials during this period, including early goal-directed therapy for severe sepsis, ${ }^{40}$ and the introduction of low tidal volume ventilation for acute lung injury, ${ }^{41}$ and an international campaign focused on sepsisrelated quality of care. ${ }^{42,43} \mathrm{~A}$ crucial question is whether these therapies are ineffective at altering the long-term natural history of severe sepsis, whether the attributable long-term mortality of severe sepsis is lower than previously believed, ${ }^{14,15}$ or whether the implementation of effective therapies has been too incomplete to affect population health. ${ }^{44}$

The results of the current study have several limitations. It focused on severe sepsis within the fee-for-service Medicare population. Although this is a population in which previous work demonstrated clear functional and cognitive disability among survivors, ${ }^{5}$ it does not include individuals with severe sepsis not yet eligible for Medicare. As such, the total population burden of severe sepsis survivorship is probably greater. Second, a widely accepted operationalization of severe sepsis for national epidemiological work was used; although this definition has been clinically validated, this is not the same as prospective assessment of patients, were such a national assessment feasible. Third, previous estimates of the associations between severe sepsis and disability showed that there was less attributable disability after severe sepsis in individuals who were already severely disabled; ${ }^{5}$ if there are more-severely disabled individuals in the U.S. national population, the estimated burden of severe sepsis survivorship may have been an overestimate. Finally, administrative data were used, and so secular trends in coding practice for specific organ dysfunctions could have influenced the results; Appendix 2 presents several sensitivity results. Overall greater attention to secondary diagnoses might account for some of the overall trend, although this would more likely represent a failure to code organ dysfunction in the past than fraudulent coding of organ dysfunction in the current period.

Severe sepsis has emerged as a dominant cause of serious illness in Medicare beneficiaries, and survivorship after severe sepsis is common. There are nearly 500,000 disabled survivors of severe sepsis yet few proven therapies or specific support programs exist to help or support their caregivers. Given the enduring cognitive, physical, and quality-of-life decrements of survivors of severe sepsis, these individuals and their families may benefit from efforts to improve and integrate their care-from the prevention of severe sepsis through its acute treatment and long-term follow-up.

\section{ACKNOWLEDGMENTS}

Conflict of Interest: The editor in chief has reviewed the conflict of interest checklist provided by the authors and has determined that the authors have no financial or any other kind of personal conflicts with this paper.

This work was supported by grant K08 HL091249 from the U.S. National Institutes of Health. We appreciate the expert programming of Letitia Shapiro, AM, at the University of Michigan.

Author Contributions: Iwashyna T. J., Cooke C. R., Wunsch H., and Kahn J. M.: Study concept and design. 
Iwashyna T. J.: Acquisition of subjects and data. Iwashyna T. J.: Analysis of data. Iwashyna T. J., Cooke C. R., Wunsch H., and Kahn J. M.: Interpretation of data. Iwashyna T. J., Cooke C. R., Wunsch H., and Kahn J. M.: Preparation of manuscript.

Sponsor's Role: The sponsor played no role in the design, methods, subject recruitment, data collections, analysis, or preparation of paper.

\section{REFERENCES}

1. Levy MM, Fink MP, Marshall JC et al. 2001 SCCM/ESICM/ACCP/ATS/ SIS International Sepsis Definitions Conference. Crit Care Med 2003;31:1250-1256.

2. Angus D, Linde-Zwirble W, Lidicker J et al. Epidemiology of severe sepsis in the United States: Analysis of incidence, outcome, and associated costs of care. Crit Care Med 2001;29:1303-1310.

3. Karlsson S, Ruokonen E, Varpula T, et al. for the Finnsepsis Study Group. Long-term outcome and quality-adjusted life years after severe sepsis. Crit Care Med 2009;37:1268-1274.

4. Winters BD, Eberlein M, Leung J et al. Long-term mortality and quality of life in sepsis: A systematic review. Crit Care Med 2010;38:1276-1283.

5. Iwashyna TJ, Ely EW, Smith DM et al. Long-term cognitive impairment and functional disability among survivors of severe sepsis. JAMA 2010;304:1787-1794.

6. Weycker D, Akhras KS, Edelsberg J et al. Long-term mortality and medical care charges in patients with severe sepsis. Crit Care Med 2003;31:23162323.

7. Lee H, Doig CJ, Ghali WA et al. Detailed cost analysis of care for survivors of severe sepsis. Crit Care Med 2004;32:981-985.

8. Langa KM, Chernew ME, Kabeto MU et al. National estimates of the quantity and cost of informal caregiving for the elderly with dementia. J Gen Intern Med 2001;16:770-778.

9. Milbrandt EB, Eldadah B, Nayfield S et al. Toward an integrated research agenda for critical illness in aging. Am J Respir Crit Care Med 2010;182:995-1003.

10. Hewitt M, Greenfield S, Stovall E, eds. From Cancer Patient to Cancer Survivor: Lost in Translation. Washington, DC: National Academies Press, 2006.

11. Cheung WY, Neville BA, Cameron DB et al. Comparisons of patient and physician expectations for cancer survivorship care. J Clin Oncol 2009;27:2489-2495.

12. Klabunde $\mathrm{CN}$, Ambs A, Keating NL et al. The role of primary care physicians in cancer care. J Gen Intern Med 2009;24:1029-1036.

13. Mullan F. Seasons of survival: Reflections of a physician with cancer. N Engl J Med 1985;313:270-273.

14. Quartin AA, Schein RMH, Kett DH et al. Magnitude and duration of the effect of sepsis on survival. JAMA 1997;277:1058-1063.

15. Wunsch H, Guerra C, Barnato AE et al. Three-year outcomes for Medicare beneficiaries who survive intensive care. JAMA 2010;303:849-856.

16. Hatten J. Medicare's common denominator: The covered population. Health Care Financ Rev 1980;2:53-64.

17. Angus DC, Wax RS. Epidemiology of sepsis: An update. Crit Care Med 2001;29:S109-S116.

18. Barnato AE, Alexander SL, Linde-Zwirble WT et al. Racial variation in the incidence, care, and outcomes of severe sepsis. Am J Respir Crit Care Med 2007;177:279-284.

19. Iwashyna TJ. Survivorship will be the defining challenge of critical care in the 21st century. Ann Intern Med 2010;153:204-205.

20. Mayr FB, Yende S, Linde-Zwirble WT et al. Infection rate and acute organ dysfunction risk as explanations for racial differences in severe sepsis. JAMA 2010;303:2495-2503.

21. Seymour CW, Iwashyna TJ, Cooke CR et al. Marital status and the epidemiology and outcomes of sepsis. Chest 2010;137:1289-1296.
22. Bone RC, Balk RA, Cerra FB et al. Definitions for sepsis and organ failure and guidelines for the use of innovative therapies in sepsis. The ACCP/ SCCM Consensus Conference Committee. American College of Chest Physicians/Society of Critical Care Medicine. Chest 1992;101:1644-1655.

23. Angus DC. The lingering consequences of sepsis: A hidden public health disaster? JAMA 2010;304:1833-1834.

24. Martin GS, Mannino DM, Eaton S et al. The epidemiology of sepsis in the United States from 1979 through 2000. N Engl J Med 2003;348:1546-1554.

25. Iwashyna TJ, Kahn JM, Hayward RA et al. Interhospital transfers among Medicare beneficiaries admitted for acute myocardial infarction at nonrevascularization hospitals. Circ Cardiovasc Qual Outcomes 2010;3:468475 .

26. Krumholz HM, Wang Y, Mattera JA et al. An administrative claims model suitable for profiling hospital performance based on 30-day mortality rates among patients with an acute myocardial infarction. Circulation 2006;113:1683-1692.

27. Epstein AJ, Rathore SS, Krumholz HM et al. Volume-based referral for cardiovascular procedures in the United States: A cross-sectional regression analysis. BMC Health Serv Res 2005;5:42.

28. Herzog AR, Wallace RB. Measure of cognitive functioning in the AHEAD Study. J Gerontol B Psychol Sci Soc Sci 1997;52B:37-48.

29. Welsh KA, Breitner JCS, Magruder-Habib KM. Detection of dementia in the elderly using telephone screening of cognitive status. Neuropsychiatry Neuropsychol Behav Neurol 1996;6:103-110.

30. Jorm AF. The Informant Questionnaire on Cognitive Decline in the Elderly (IQCODE): A review. Int Psychogeriatr 2004;16:275-293.

31. Lazosky A, Young GB, Zirul S et al. Quality of life after septic illness. J Crit Care 2010;25:406-412.

32. Wilhelms SB, Huss FR, Granath G et al. Assessment of incidence of severe sepsis in Sweden using different ways of abstracting International Classification of Diseases codes: Difficulties with methods and interpretation of results. Crit Care Med 2010;38:1442-1449.

33. Schweickert WD, Pohlman MC, Pohlman AS et al. Early physical and occupational therapy in mechanically ventilated, critically ill patients: A randomised controlled trial. Lancet 2009;373:1874-1882.

34. Girard TD, Jackson JC, Pandharipande PP et al. Delirium as a predictor of long-term cognitive impairment in survivors of critical illness. Crit Care Med 2010;38:1513-1520.

35. Dellinger RP, Levy MM, Carlet JM et al. Surviving Sepsis Campaign: International guidelines for management of severe sepsis and septic shock: 2008. Crit Care Med 2008;36:296-327.

36. Kumar A, Roberts D, Wood KE et al. Duration of hypotension before initiation of effective antimicrobial therapy is the critical determinant of survival in human septic shock. Crit Care Med 2006;34:1589-1596.

37. Khan MM. Immunosuppressive agents. In: Kahn MM, eds. Immunopharmacology. New York, NY: Springer, 2009, pp 1-19.

38. Bernstein AB, Hing E, Moss AJ et al. Health Care in America: Trends in Utilization. Hyattsville, MD: National Center for Health Statistics, 2003.

39. Song Y, Skinner J, Bynum J et al. Regional variations in diagnostic practices. N Engl J Med 2010;363:45-53.

40. Rivers E, Nguyen B, Havstad S et al. Early goal-directed therapy in the treatment of severe sepsis and septic shock. N Engl J Med 2001;345:13681377.

41. Acute Respiratory Distress Syndrome Network. Ventilation with lower tidal volumes as compared with traditional tidal volumes for acute lung injury and the acute respiratory distress syndrome. $\mathrm{N}$ Engl J Med 2000;342:1301-1308.

42. Slade E, Tamber PS, Vincent JL. The Surviving Sepsis Campaign: Raising awareness to reduce mortality. Crit Care 2003;7:1-2.

43. Dellinger RP, Carlet JM, Masur H et al. Surviving Sepsis Campaign guidelines for management of severe sepsis and septic shock. Crit Care Med 2004;32:858-873.

44. Ferrer R, Artigas A, Levy MM et al. Improvement in process of care and outcome after a multicenter severe sepsis educational program in Spain. JAMA 2008;299:2294-2303. 


\section{APPENDICES}

\section{Example of Demographic Decomposition}

To understand the relative contributions to the changing rates of severe sepsis survivorship, the following approach is used. First, consider women aged 65 to 69. The following values were calculated for 1996 from the Medicare data:

- POP: the number of Medicare recipients in this group

- HOSP: their age- and sex-specific rate of hospitalization with infection

- DYSFUNC: the aggregate rate of organ dysfunction per hospitalization

- SURVIVE: the aggregate 3-year survival rate for individuals with severe sepsis

The total number of 3-year survivors in this age group of women is mathematically equivalent to POP $\times$ HOSP $\times$ DYSFUNC $\times$ SURVIVE. Adding subscripts to indicate the reference year, the sex, and the age group, then the number of 3-year survivors96,w,65-69 $=$ POP $_{96, \mathrm{~W}, 65-69} \times \mathrm{HOSP}_{96, \mathrm{~W}, 65-69} \times \mathrm{DYSFUNC}_{96} \times \mathrm{SURVIVE}_{96}$

The total number of 3-year survivors is the sum over both sexes and all age groups of these age- and sex-specific numbers of 3-year survivors.

To calculate the effect of population-aging between 1996 and 2005, what would have happened if $\mathrm{POP}_{96, \mathrm{~W}, 65-69}$ were replaced in the above equations by $\mathrm{POP}_{2005, \mathrm{w}, 65-69}$ is considered. HOSP $96, \mathrm{w}, 65-69$, DYSFUNC $_{96}$, and SURVIVE 96 are held constant, but the sum is recalculated. The ratio of this simulated number for 2005 to the actual number of 3-year survivors in 1996 was 1.057 . Thus the change in the age distribution accounted for a $5.7 \%$ increase in the number of 3-year survivors over that period, as presented in Figure 4.

A similar logic could be used changing only the year-specific rates for each of the other three terms. This was done to produce Figure 4.

\section{Sensitivity Analyses to Alternative Severe Sepsis Definitions}

In 2002, a new International Classification of Diseases, Ninth Revision, Clinical Modification, code for "severe sepsis" was introduced. Excluding cases of severe sepsis with this explicit code had little effect on these results, because most such hospitalizations also had codes for a specific infection and at least one acute organ dysfunction. The analyses were further replicated with alternative definitions of severe sepsis that excluded each organ dysfunction to ensure that unspecified changes in coding practice for other conditions did not determine the results. (That is, a definition of severe sepsis was considered to be an infection plus an organ dysfunction other than, for example, hepatic injury.) Severe sepsis survivorship rose significantly in all such sensitivity tests, although less so if acute kidney injury was excluded as a severe sepsis-defining organ dysfunction.

This table is organized as follows. Each column of the table presents the results of the analyses as they occurred under alternative definitions of severe sepsis. The first column, for reference, provides the results presented in the body of the manuscript. Important findings are in the italicized rows - they show, for example, a consistent percentage increase in the number of 3-year survivors across the definitions. The rows entitled "Contributions to 3-year survivorship change" parallel the analysis summarized in Figure 4.

Finally, results for 5-year survival, as is commonly used in oncology, are presented here as well and show a consistent pattern.

Sensitivity Analyses Excluding Codes for..

\begin{tabular}{|c|c|c|c|c|c|c|c|c|}
\hline Analytical Variable & $\begin{array}{c}\text { Full } \\
\text { Definition }\end{array}$ & \\
\hline $\begin{array}{l}\text { New 3-year survivors, } 1999 \\
\text { (got sepsis in 1996), n }\end{array}$ & 102,767 & 102,767 & 73,051 & 87,398 & 83,898 & 102,332 & 82,327 & 73,260 \\
\hline $\begin{array}{l}\text { New 3-year survivors, } 2008 \\
\text { (got sepsis in 2005), n }\end{array}$ & 225,251 & 207,410 & 164,540 & 203,084 & 188,885 & 223,797 & 124,560 & 189,416 \\
\hline Change, 2008 vs 1999, \% & 219 & 202 & 225 & 232 & 225 & 219 & 151 & 259 \\
\hline $\begin{array}{l}\text { New 5-year survivors, } 2008 \\
\text { (got sepsis in 2003), n }\end{array}$ & 121,029 & 119,756 & 85,743 & 108,803 & 98,814 & 12,0262 & 76,004 & 97,170 \\
\hline Change, 2008 vs 2001, \% & 179 & 177 & 183 & 187 & 180 & 178 & 138 & 199 \\
\hline Incident cases 1996, n & 387,330 & 387,330 & 289,614 & 341,586 & 320,965 & 384,128 & 284,144 & 240,175 \\
\hline Incident cases 2003, n & 656,194 & 645,379 & 488,511 & 594,821 & 544,488 & 650,126 & 376,227 & 467,504 \\
\hline Incident cases 2005, n & 791,809 & 689,826 & 590,572 & 722,414 & 668,707 & 783,373 & 413,788 & 597,456 \\
\hline
\end{tabular}




\section{(Contd.)}

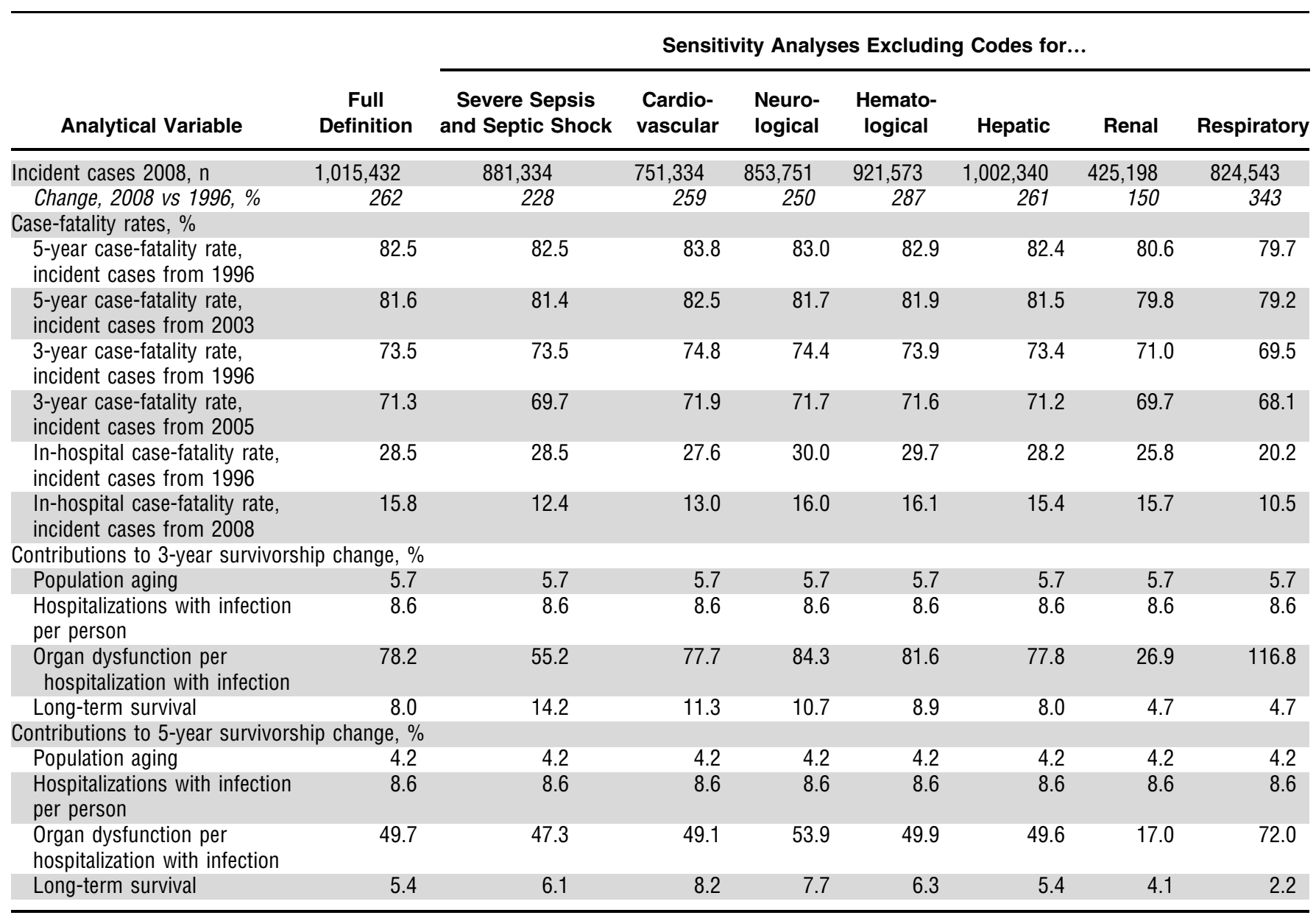

